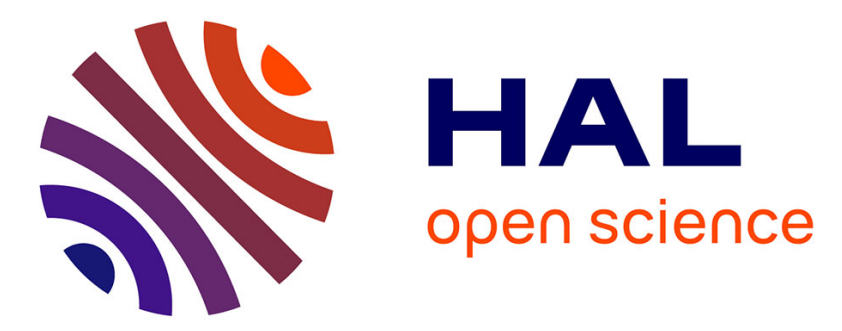

\title{
Croissance et développement du Pachyrhizus erosus (Urban) I. - Effets de l'acide gibbérellique et du chlorure de chlorocholine en jours courts
}

Claude Zinsou, Alfred Venthou-Dumaine, Gérard Vansuyt, Camille Constant

\section{- To cite this version:}

Claude Zinsou, Alfred Venthou-Dumaine, Gérard Vansuyt, Camille Constant. Croissance et développement du Pachyrhizus erosus (Urban) I. - Effets de l'acide gibbérellique et du chlorure de chlorocholine en jours courts. Agronomie, 1987, 7 (9), pp.677-683. hal-00885041

\section{HAL Id: hal-00885041 https://hal.science/hal-00885041}

Submitted on 1 Jan 1987

HAL is a multi-disciplinary open access archive for the deposit and dissemination of scientific research documents, whether they are published or not. The documents may come from teaching and research institutions in France or abroad, or from public or private research centers.
L'archive ouverte pluridisciplinaire HAL, est destinée au dépôt et à la diffusion de documents scientifiques de niveau recherche, publiés ou non, émanant des établissements d'enseignement et de recherche français ou étrangers, des laboratoires publics ou privés. 


\title{
Croissance et développement du Pachyrhizus erosus (Urban) I. - Effets de l'acide gibbérel- lique et du chlorure de chlorocholine en jours
} courts

\author{
Claude ZINSOU, Alfred VENTHOU-DUMAINE \& Gérard VANSUYT \\ avec la collaboration technique de Camille CONSTANT
}

I.N.R.A., Laboratoire de Physiologie et Biochimie végétales, Centre de Recherches des Antilles et de la Guyane, B.P. 1232, F 97184 Pointe-à-Pitre Cedex (F.W.I., Guadeloupe). choline aux doses de 1000 et 5000 p.p.m. (CCC 1000 et CCC 5000 ) ont été étudiés au champ sur la croissance, la tubérisation et la floraison du dolique tubéreux, légumineuse tropicale à graines et à tubercule. GA stimule la mise en place de la partie aérienne, induit le grossissement précoce et rapide du tubercule et inhibe la floraison et la fructification. Le CCC a un effet favorable sur la floraison et la fructification mais inhibe partiellement le grossissement du tubercule. Seules les plantes traitées au GA 50 présentent une accumulation de matière sèche totale par plante en fin de cycle supérieure à celle obtenue avec les autres traitements. L'analyse de la répartition finale de la matière sèche totale révèle que la part affectée au fonctionnement de la partie aérienne végétative (tiges et feuilles) est pratiquement constante. Les substances de croissance ont induit essentiellement une répartition différente des assimilats totaux stockés entre les deux organes de réserve : gousses et tubercule.

Mots clés additionnels : Tubérisation, floraison, organes de réserve, légumineuse tropicale, dolique tubéreux. (GA3) and chlorocholine chloride (CCC) under short-day conditions.

The effects of gibberellic acid at 50 and 200 p.p.m. (GA 50 and GA 200) and chlorocholine chloride 1000 and 5000 (CCC 1000 and CCC 5000$)$ were investigated in the field on growth, flowering and tuberization of yam bean, a tropical legume producing grain and tubers. GA enhanced the development of the aerial parts and tubers but inhibited flowering. $\mathrm{CCC}$ favoured flowering and pod formation but reduced tuber development. Only GA 50 plants presented dry matter accumulation per plant higher than that obtained with other treatments. Analysis of matter distribution revealed that the \% share allocated to aerial parts (leaves and stems) and to storage organs (pods and tubers) was almost constant. The effects of growth substances essentially resulted in a different distribution of dry matter between the two storage organs, pods and tubers.

Additional key words : Tuberization, flowering, storage organs, tropical legume, yam bean.

\section{INTRODUCTION}

Le dolique tubéreux ou mexican yam bean, Pachyrhizus erosus (Urban), est une légumineuse à graines et à tubercules de la famille des papilionacées. Originaire du Mexique où il était déjà connu des civilisations précolombiennes, il est aujourd'hui largement disséminé dans les régions tropicales en Chine, Inde, Indonésie, aux Philippines (SCHROEDER, 1967, SRI-
VASTAVA et al., 1973), sauf en Afrique où il existe un autre dolique tubéreux, african yam bean, Sphenostylis stenocarpa (Harms), produisant aussi des graines et des tubercules (OKIGBO, 1973). Le P. erosus est peu connu et peu cultivé dans la région caraïbe où les gousses et les tubercules pourraient fournir des glucides et des protéines (MARTA EvANS et al., 1977 ; ZINSOU et al., 1987) à bon marché dans l'alimentation humaine et animale grâce à l'aptitude de la plante à 
fixer directement l'azote atmosphérique pour ses propres besoins (LAMAZE et al., 1985).

La partie aérienne du Pachyrhizus contient 0,12 à 0,43 p. 100 de roténone par rapport à la matière sèche (HANSBERRY et al., 1945), ce qui rend les graines matures, riches en protéines $(26,2$ p. 100$)$ et en lipides (27,2 p. 100), impropres à la consommation. Cependant les gousses immatures, d'environ 2 semaines, sont consommées cuites, sans dommage pour l'homme, comme haricots verts en Amérique Centrale et aux Philippines (SCHROEDER, 1967). Le tubercule, exempt de roténone, contient du glucose, fructose et de l'amidon, digestible sans cuisson. Il est équilibré en acides aminés (MARTA EVANS et al., 1977) et peut être consommé cru ou cuit. Outre son intérêt alimentaire grâce aux tubercules et gousses immatures, la partie verte, riche en azote (15 à $20 \mathrm{p} .100$ de protéines) peut être utilisée comme fourrage ou comme engrais vert pour améliorer les propriétés physiques et chimiques des sols tropicaux grâce à l'apport de matière et de l'azote sous forme organique (CORTES, 1970).

Nos premiers travaux, non publiés, montrent que, quelle que soit l'époque de semis et malgré une tubérisation précoce qui est initiée entre la $4^{\mathrm{e}}$ et la $6^{\mathrm{e}}$ semaine, la floraison, la formation et le remplissage des gousses d'une part, le développement actif du tubercule d'autre part, se déroulent en jours courts ou décroissants. La photophase maximale en Guadeloupe est de 13 heures. La présence de 2 sites de réserve à remplir simultanément, conférant à cette plante le caractère d'un modèle biologique intéressant, entraîne une compétition pour l'utilisation et le stockage des assimilats. Dans le cas où seule la production du tubercule est recherchée, une pratique courante au Mexique consiste en l'ablation à un stade précoce de toute tige florifère apparaissant sur la plante (ZEPEDA, 1971). La plante étant très photosensible, les conditions photopériodiques déterminent le port (port de haricot nain en jours courts, port lianescent et volubile entre 12 et $13 \mathrm{~h}$ ) et interviennent dans son fonctionnement. C'est ainsi que la croissance en jours inférieurs à 12 heures suffit à orienter précocement la physiologie de la plante vers le remplissage du tubercule en réduisant le développement de la partie végétative aérienne.

Ce travail rapporte pour des plantes cultivées, en jours courts, les effets de l'acide gibbérellique et du chlorure de chlorocholine, 2 régulateurs de croissance susceptibles de modifier les caractères de croissance et de distribution de matière entre les différents organes de la plante.

\section{MATÉRIEL ET MÉTHODES}

\section{A. Matériel}

Le matériel végétal utilisé est le Pachyrhizus erosus (yam bean ou dolique tubéreux) légumineuse tropicale tubérifère, originaire du Mexique. Ses graines ont été généreusement fournies par le Dr STEELE de l'I.I.T.A. d'Ibadan (Nigéria) et multipliées sur place.

\section{B. Méthodes}

L'expérimentation a été établie au champ sur par- celles de sol ferrallitique et conduite d'octobre à février. Les graines ont été semées tous les $60 \mathrm{~cm}$ sur des billons espacés de $1 \mathrm{~m}$. Chaque traitement comporte 250 plantes homogénéisées avant le début des prélèvements. Les plantes n'ont pas été inoculées. Le rhizobium nodulant le Pachyrhizus fait partie du groupe «cowpea ». Tous les essais effectués jusqu'ici montrent qu'il est efficient et présent dans le sol.

La fertilisation est localisée et apportée sous forme d'engrais potassiques et phosphorés, mélangés sur environ $30 \mathrm{~cm}$ de diamètre autour du pied de la plante. Aucun apport d'azote n'a été effectué. Cependant le Pachyrhizus semble avoir profité de la fumure azotée de la culture précédente. Ce qui pourrait expliquer la faible nodulation observée sur tous les traitements sauf en présence de GA 50. Le phosphate a été apporté sous forme de scories de déphosphoration à raison de 70 unités de $\mathrm{P}_{2} \mathrm{O}_{5}$ à l'hectare, les $2 / 3$ au moment du semis et le reste au stade 3 feuilles en même temps que le potassium à raison de 120 unités de $\mathrm{K}_{2} \mathrm{O}$ à l'hectare. Les scories ont l'avantage de réaliser en même temps un apport de chaux et de magnésium sur les sols ferrallitiques acides.

Les plantes sont traitées avec des solutions d'acide gibbérellique $\mathrm{GA}_{3}$ à la dose de 50 et 200 p.p.m. (GA 50 et GA 200) ou de chlorure de chlorocholine, à la dose de 1000 et 5000 p.p.m. (CCC 1000 et CCC 5 000) par pulvérisation sur toute la partie aérienne. Les plantes témoins sont pulvérisées avec de l'eau. Les traitements sont effectués tous les 15 jours à partir de la $5^{\mathrm{e}}$ semaine et après le premier prélèvement. Cinq traitements ont été réalisés. Des prélèvements de 10 plantes par traitement, tirées au hasard, ont été effectués toutes les semaines entre la $4^{\mathrm{e}}$ et la $10^{\mathrm{e}}$ semaine puis tous les 15 jours pour déterminer le nombre de feuilles et les matières sèches des principaux organes.

Les traitements statistiques des résultats ont été effectués à l'aide de l'analyse de variance non paramétrique de KRUSKAL-WALLIS pour la comparaison de plusieurs populations et à l'aide du test $T$ par paires pour la comparaison de chaque traitement au témoin (DELECOLlE \& SiMON, 1976).

\section{RÉSULTATS}

\section{A. Effets des traitements sur la partie aérienne}

\section{Nombre de feuilles}

Le nombre de feuilles émises au cours de la croissance du Pachyrhizus varie suivant les traitements (fig. 1).

L'acide gibbérellique stimule l'émission des feuilles. Sur les plantes traitées au GA 50 la courbe d'émission des feuilles est linéaire jusqu'à la fin de la $12^{\mathrm{e}}$ semaine, puis apparaît un léger fléchissement (fig. 1A). Lorsqu'on compare les plantes témoins et les plantes traitées, l'effet du traitement au GA 50 analysé par le test $T$ par paires est significatif dès la $9^{\mathrm{e}}$ semaine jusqu'à la fin du cycle au niveau $\alpha=0,01$. En ce qui concerne le GA 200 l'effet ne devient significatif au niveau $\alpha=0,05$ qu'à partir de la formation des gousses au moment où cette dose 


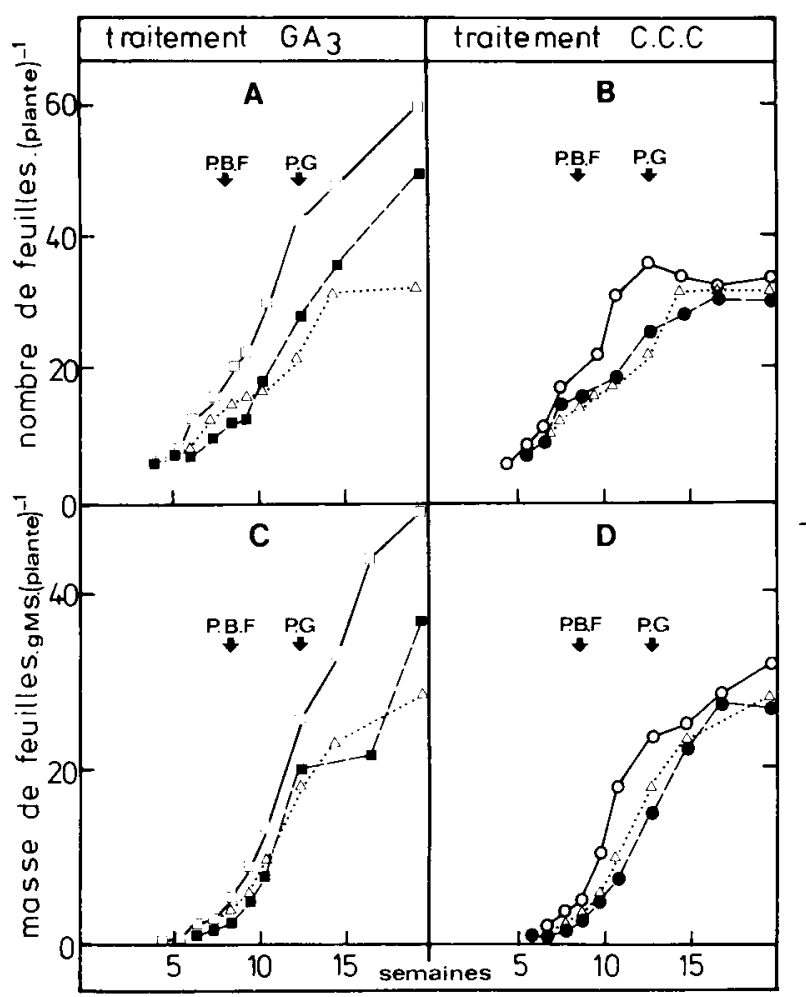

Figure 1

Evolution du nombre de feuilles par plante $(A$ et $B$ ) et de la matière sèche des limbes par plante $(C$ et $D$ ) au cours de la croissance des plantes de dolique tubéreux sous différents traitements de phyto-

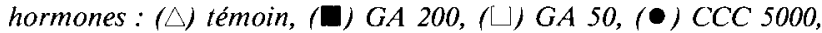
(O) CCC 1000. Chaque point représente la moyenne de 10 plantes. $P B F$ représente l'apparition des premiers bourgeons floraux et $P G$ le début de formation des gousses.

Changes in leaf number per plant $(A, B)$ and in dry weight matter of leaves per plant $(C, D)$ during the growth of yam bean under different growth regulator treatments: ( $\triangle$ ) control, (⿴) GA 200, (1) $G A 50,(\bullet) C C C 5000,(0) C C C 1000$. Each point is the average of 10 plants. PBF indicates the appearance of first floral buds and $P G$ the beginning of pod set.

provoque l'avortement des fleurs et une mauvaise nouaison.

En fin de cycle le nombre de feuilles sur les plantes traitées au GA 50 est pratiquement le double de celui observé sur les plantes témoins.

Les plantes traitées au CCC 5000 présentent un nombre de feuilles supérieur à celui observé sur les plantes témoins entre la $8^{\mathrm{e}}$ et la $12^{\mathrm{e}}$ semaine. Cet effet est significatif au niveau $\alpha=0,05$. Pendant cette période, le CC 1000 induit un rythme d'émergence des feuilles plus rapide. Cependant on constate que l'écart entre les nombres de feuilles des plantes témoins et les plantes traitées au CCC se réduit et n'est pas significatif au niveau $\alpha=0,1$ dès que s'installent la formation et le remplissage des gousses (fig. 1B). Le rythme rapide d'émergence des feuilles induit par le CCC 1000 entre la $8^{\mathrm{e}}$ et la $19^{\mathrm{e}}$ semaine ne se traduit pas en fin de cycle par un nombre significativement plus élevé de feuilles sur les plantes traitées.

\section{Matière sèche des limbes}

Les traitements au GA 200 et au CCC 5000 n'affectent pas de façon significative l'accumulation de matière sèche dans les limbes des plantes traitées lorsqu'on les compare aux témoins. A ces doses, le GA et le CCC ont peu d'effet (fig. 1C et D). En revanche, les plantes traitées au GA 50 et au CCC 1000 présentent une évolution identique de la matière sèche dans leurs limbes. Les quantités de matière accumulées sont significativement supérieures au niveau $\alpha=0,05$ à celles obtenues avec les plantes traitées au GA 200 et au CCC 5000 dès le début des traitements et jusqu'à la fin de la $12^{\mathrm{e}}$ semaine. A partir de ce moment la courbe d'accumulation de la matière sèche sous traitement de CCC 1000 s'infléchit au point qu'à la $20^{\mathrm{e}}$ semaine on retrouve pratiquement les mêmes valeurs pour les plantes témoins et pour celles qui sont traitées au CCC 1000 , CCC 5000 et GA 200. Seules les plantes traitées au GA 50 continuent après la $12^{\mathrm{e}}$ semaine à accumuler de la matière sèche dans les limbes et s'écartent ainsi des autres traitements (fig. $1 \mathrm{C}$ et D).

\section{Matière sèche des tiges et pétioles}

L'accumulation importante de matière dans les tiges et dans les pétioles débute vers la $8^{\mathrm{e}}$ semaine après les premières manifestations de la floraison. Elle est en partie liée à la mise en place de nouveaux organes que sont les nombreuses hampes florales. Les courbes d'évolution de la matière sèche dans les tiges, les pétioles et les hampes s'infléchit à partir de la $14^{\mathrm{e}}$ semaine. Le cycle de la plante se poursuit alors, au niveau de la partie aérienne, par le développement des organes reproducteurs.

En présence du CCC on constate que l'accroissement de la matière sèche dans les plantes témoins est intermédiaire entre ceux des plantes traitées au CCC 1000 et au CCC 5000 . Mais en fin de cycle les matières sèches accumulées dans les plantes traitées sont supérieures à celle observée chez les témoins (fig. 2B). Le CCC favorise le développement des organes florifères.

C'est avec le GA 50 que nous obtenons la plus forte accumulation de matière dans les tiges et les pétioles et les hampes florales au bout de 20 semaines (fig. 2A). Elle est due à l'allongement des entrenœuds et à la formation de tiges secondaires lianescentes qu'on observe habituellement en jours longs. Les plantes traitées au GA 200 montrent un ralentissement dans la croissance des tiges et pétioles entre la $12^{\mathrm{e}}$ et la $16^{\mathrm{e}}$ semaine pendant la floraison, mais cette dernière reprend ensuite en l'absence de fructification consécutive à l'avortement des fleurs.

\section{B. Effets des régulateurs de croissance sur la partie souterraine : racine et tubercule}

Le Pachyrhizus présente une seule racine pivotante (rarement 2) qui tubérise. Des radicelles partent de la racine principale et portent habituellement les nodosités dont les matières sèches faibles, n'ont pas été estimées. L'induction de la tubérisation est précoce chez le Pachyrhizus. Un début de renflement de la racine peut être perçu entre la $4^{\mathrm{e}}$ et la $6^{\mathrm{e}}$ semaine après le semis. Les traitements aux régulateurs de croissance ont débuté pendant la période d'induction et aucune inhibition n'a été constatée. Les résultats ne se rap- 


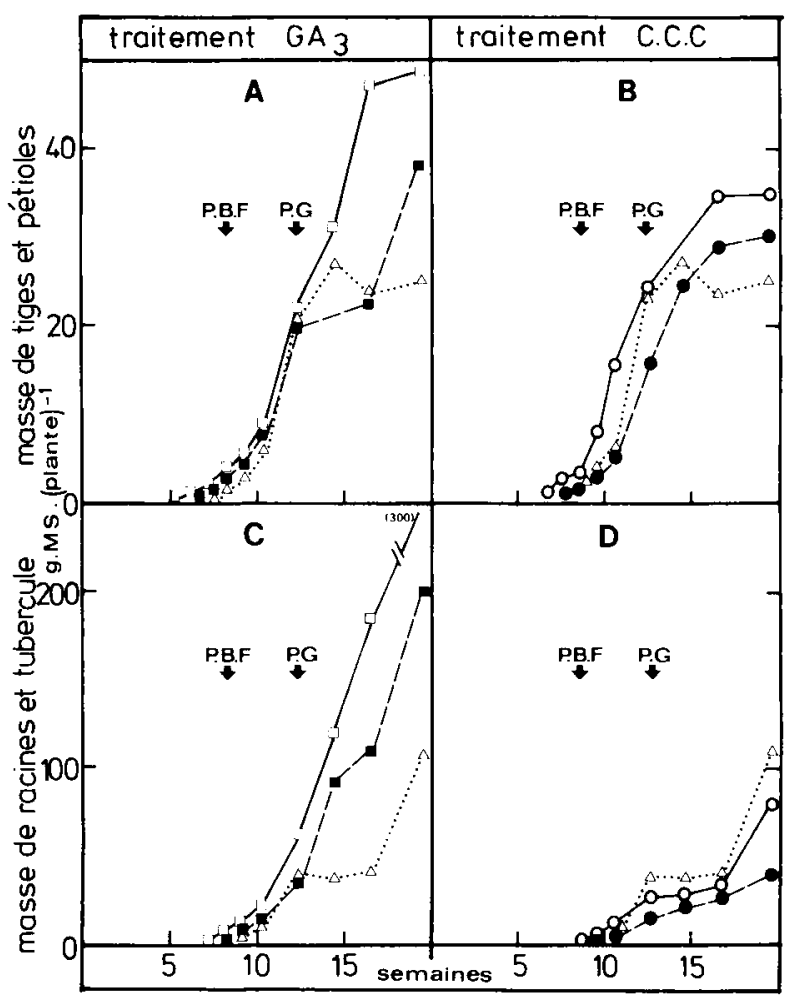

Figure 2

Matière sèche des tiges et pétioles par plante $A, B$ et des racinestubercules par plante $C, D$ au cours de la croissance du dolique tubéreux sous différents traitements de phytohormones: $(\Delta)$

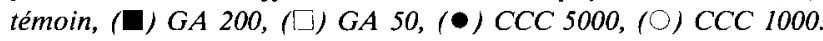
Chaque point est la moyenne de 10 plantes. PBF : apparition des premiers bourgeons floraux ; $P G$ : début de formation des gousses.

Changes in dry matter of stems and petioles per plant $(A, B)$ and tuberous root per plant $(C, D)$ during the growth of yam bean under different growth regulator treatments: $(\triangle)$ control,

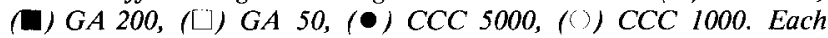
point is the average of 10 plants. PBF : appearance of first floral buds ; $P G$ : beginning of pod set.

portent donc qu'aux effets de ces facteurs de croissance sur le grossissement du tubercule.

Quelle que soit la dose utilisée, l'acide gibbérellique, sous les conditions de jours courts, favorise le développement du tubercule alors que le CCC l'inhibe partiellement. Le traitement est plus efficace à la dose de 50 p.p.m. qu'à la dose de 200 p.p.m. pour l'acide gibbérellique (fig. $2 \mathrm{C}$ ). A la $20^{\mathrm{e}}$ semaine les matières sèches des tubercules des plantes traitées au GA 50 et GA 200 sont respectivement 2,7 et 1,8 fois plus élevées que celles rencontrées dans les tubercules des plantes témoins. Avec le CCC 1000 et CCC 5000 elles sont respectivement 0,78 et 0,37 fois celles des témoins (fig. 2D).

Entre la $12^{\mathrm{e}}$ et la $16^{\mathrm{e}}$ semaine, la courbe d'accumulation de la matière dans les tubercules présente un plateau sur les plantes témoins et celles traitées au CCC 1000 . Il est moins accusé avec le GA 200 et absent avec le GA 50. Ce plateau est le reflet des relations de compétition qui s'instaurent entre le puits aérien (gousses et graines) et le puits souterrain (tubercule). En effet, l'accumulation de la matière sèche dans le tubercule ralentit fortement au moment de la formation des gousses. Elles ne reprend que pendant le remplissage de ces organes. La faible nouaison précédée d'un échelonnement de la floraison chez les plantes traitées à l'acide gibbérellique se traduit par l'absence de ce plateau; le seul organe se remplissant est alors le tubercule.

L'analyse de variance non paramétrique de KRUSKAL-WALLIS sur les poids des matières sèches des tubercules révèle qu'à partir de la $8^{\mathrm{e}}$ semaine, l'hypothèse de l'homogénéité des populations peut être rejetée au niveau $\alpha=0,01$. En comparant chaque traitement au témoin, l'effet promoteur du grossissement $\mathrm{du}$ tubercule de l'acide gibbérellique est significatif au niveau $\alpha=0,01$ pour GA 50 et au niveau $\alpha=0,05$ pour GA 200 de la $8^{\mathrm{e}}$ semaine jusqu'à la fin du cycle. En ce qui concerne l'effet inhibiteur du CCC, il est significatif au niveau $\alpha=0,05$ pour le CCC 5000 et CCC 1000.

A la fin du cycle de la plante ( 5 mois après semis) le poids frais moyen du tubercule (chaque plante produit un tubercule) déterminé à partir d'un échantillon de 50 plantes par traitement est : $(1200 \pm 200)$ g pour le témoin, $(430 \pm 130) \mathrm{g}$ et $(900 \pm 250) \mathrm{g}$ respectivement pour les CCC 5000 et CCC $1000 ;(1800 \pm$ $300) \mathrm{g}$ et $(3500 \pm 800) \mathrm{g}$ respectivement pour le GA 200 et le GA 50.

\section{Effets des régulateurs de croissance sur PA/PS}

PA/PS représente le rapport des matières sèches des parties aériennes et des parties souterraines.

Les courbes de la fig. 3 montrent les effets des 2 substances sur l'évolution du PA/PS. Le comportement des plantes traitées à l'acide gibbérellique est très différent de celui des plantes pulvérisées avec du chlorure de chlorocholine.

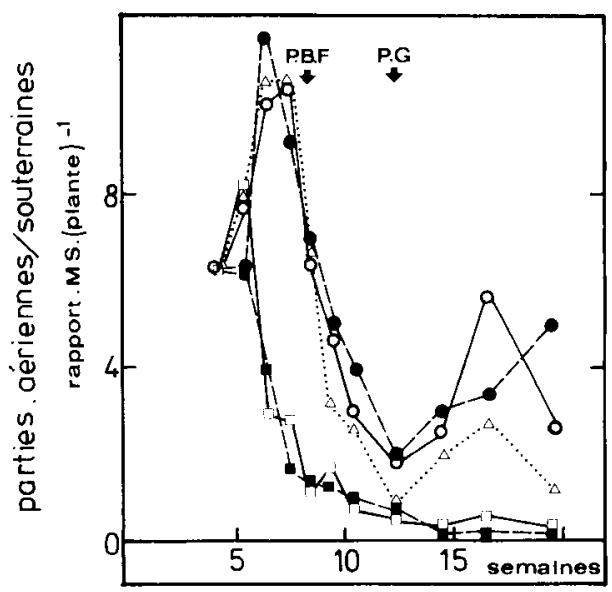

Figure 3

Evolution du rapport des matières sèches des parties aérienne et souterraine en fonction des traitements appliqués aux plantes du dolique tubéreux au cours de la croissance. GA 200 (四), $G A 50(\square), \quad C C C 1000(0), C C C 5000(\bullet)$, témoin $(\triangle)$. $P B F$ : apparition des premiers bourgeons floraux ; $P G$ : début de formation des gousses.

Changes in ratio of dry matter of aerial parts over underground parts according to the treatments applied to plants of yam bean

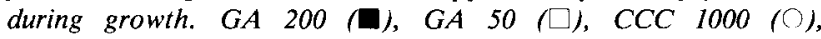
$C C C 5000(\bullet)$, control $\triangle \triangle$. PBF : appearance of first floral buds; $P G$ : beginning of pod set.

Quelle que soit la dose utilisée le rapport PA/PS des plantes traitées au GA décroît dès la $6^{\mathrm{e}}$ semaine jusqu'à la $14^{\mathrm{e}}$ semaine puis se stabilise. Il passe de 6 à 
la $6^{\mathrm{e}}$ semaine à une valeur inférieure à 1 après la $12^{\mathrm{e}}$ semaine. La répartition de la matière sèche se fait donc progressivement au profit des parties souterraines.

Les plantes traitées au CCC présentent une courbe d'évolution du PA/PS analogue à celle qui est obtenue avec le témoin. On peut y distinguer plusieurs phases :

- Le rapport PA/PS s'élève : on assiste à une croissance active de la partie aérienne pendant que le grossissement du tubercule est encore faible.

- Il passe par un maximum puis décroît jusqu'à la $12^{\mathrm{e}}$ semaine. Cette phase de décroissance est analogue à celle rencontrée sur les plantes traitées au GA. C'est la phase active de grossissement du tubercule.

- Entre la $12^{\mathrm{e}}$ et la $20^{\mathrm{e}}$ semaine, apparaît un second maximum de PA/PS qui se situe à l'époque de la formation et du remplissage des gousses. Sur les plantes témoins ou traitées au CCC 1000 , il correspond au début de la reprise du grossissement du tubercule arrêté entre la $12^{\mathrm{e}}$ et la $16^{\mathrm{e}}$ semaine (fig. 2D). Pendant la phase de montée du pic, les assimilats sont préférentiellement utilisés pour la formation des gousses.

- Si on compare les effets des 2 substances, on constate :

a) Une stimulation précoce du grossissement du tubercule chez les plantes traitées à l'acide gibbérellique. En fin de cycle, l'obtention d'un rapport PA/PS inférieur à 1 révèle un remplissage des tubercules plus efficace que sous les autres traitements. L'absence de pic entre la $12^{\mathrm{e}}$ et la $20^{\mathrm{e}}$ semaine est le reflet d'une floraison réduite doublée d'une mauvaise nouaison en présence de GA. Ceci a pour conséquence la réduction ou la suppression de la capacité de stockage du $2^{\mathrm{e}}$ puits qu'auraient constitué les graines.

b) En revanche, le $\mathrm{CCC}$ favorise la formation de gousses et de graines au détriment du tubercule. On peut dire alors que le CCC inhibe partiellement le grossissement du tubercule.

\section{Effets sur la floraison et la fructification}

Ils se manifestent au moment de l'apparition des premiers bourgeons floraux et sur l'ensemble du processus floraison-fructification. Les premiers organes floraux apparaissent entre la $8^{\mathrm{e}}$ et la $9^{\mathrm{e}}$ semaine pour tous les traitements sauf pour le GA 200. Elle est retardée d'environ 4 semaines dans ce dernier cas.

Par rapport au témoin, le traitement au CCC ne hâte pas l'anthèse des premières fleurs des hampes florales mais influe sur le nombre des gousses et des grains formés en améliorant la nouaison. Pour des poids voisins de matières sèches accumulées dans les tiges et feuilles, les rendements en gousses par plante sont significativement différents. A la récolte le rapport, poids sec des gousses sur celui des feuilles et tiges, est 2,6 pour le $\mathrm{CCC} 1000,2,75$ pour le CCC 5000 et 1,8 pour le témoin. Comme on peut le constater, l'accumulation de matière dans la partie aérienne à partir de la $12^{\mathrm{e}}$ semaine est due essentiellement à la formation et au remplissage des gousses (fig. 4B).

En ce qui concerne l'acide gibbérellique on observe un effet inhibiteur partiel sur la formation des organes

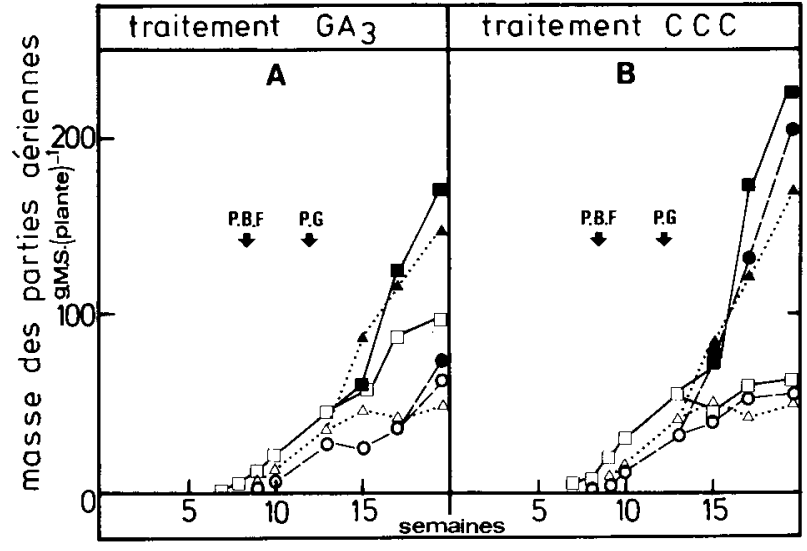

Figure 4

Matière sèche des parties aériennes sous différents traitements en $A$, GA $50(\square$ ou $\square), G A 200(\bigcirc$ ou $\bullet)$, témoin $(\triangle$ ou $\Delta)$; en $B$, CCC 1000 ( $\square$ ou $\mathbf{\square}$ ), CCC 5000 (O ou $\bullet$ ). A partir de la formation des gousses $P G$, chaque courbe se divise en deux. L'écart entre les deux courbes représente la matière sèche des gousses.

Dry matter of aerial parts under different treatments : in $A, G A 50$ $(\sqcup$ or $\mathbf{\square}), G A 200$ ( $O$ or $\bullet$ ), control $(\triangle$ or $\Delta$ ); in B, CCC 1000 $\square$ or $\square)$, CCC 5000 ( $\bigcirc$ or $\bullet$ ). From pod set $(P G)$, each curve divided into two curves. The difference between them represents the dry matter of pods.

floraux avec le GA 50, l'avortement et une mauvaise nouaison avec le GA 200 . A la $20^{\mathrm{e}}$ semaine le même rapport défini comme précédemment est de 0,6 pour le GA 50 et 0,1 pour le GA 200. La fructification chez le dolique tubéreux est pratiquement éliminée avec l'acide gibbérellique à la dose de 200 p.p.m. (fig. 4A).

\section{E. Effets des substances de croissance sur la matière sèche totale et sa répartition dans les différents organes}

A la fin de la $20^{\mathrm{e}}$ semaine représentant la date de récolte des tubercules, on observe que les plantes témoins et celles traitées au GA 200 et au CCC ont accumulé pratiquement les mêmes quantités de matière sèche. En revanche, les plantes traitées au GA 50 ont présenté des poids de matière sèche supérieurs à ceux des plantes témoins avec un écart atteignant 80 p. 100.

La répartition de la matière sèche stockée dans les feuilles et tiges, fleurs et fruits, racines et tubercule, a été analysée à la récolte (tabl. 1). On s'aperçoit que le pourcentage, relatif aux tiges et feuilles, se situe autour de 20 p. 100 quel que soit le traitement sauf en présence de GA 200 où il atteint 28 p. 100. La fraction de matière stockée dans ces organes est donc stable ; il en est de même du pool total de matière distribuée dans les autres parties, qui est voisin de 75 p. 100 de la matière sèche totale de la plante. Alors que la répa-tition semble à peu près équilibrée entre le puits aérien (37 p. 100) et le puits souterrain (42 p. 100) chez les plantes témoins, le CCC favorise le premier (52 p. 100 sous CCC 1000 et 61 p. 100 sous CCC 5 000) en provoquant une floraison groupée et une nouaison efficace. La tendance est inversée avec les plantes traitées au GA où l'accumulation de la matière sèche se fait préférentiellement dans le tubercule (64 p. 100 sous GA 50 et 71,7 p. 100 sous 
TABLEAU 1

Répartition en pourcentage, à la fin de la $20^{e}$ semaine, de la matière sèche de la plante allouée aux 3 compartiments suivants tiges + feuilles, racines et tubercule, gousses

$\%$ Distribution, at the end of 20 weeks, of plant drv matter allocated to the following three compartments: pods, leaves and stems, tuberous root.

\begin{tabular}{lccccc}
\hline \multicolumn{1}{c}{ Traitements } & Témoin $\begin{array}{r}\text { CCC } \\
1000\end{array}$ & $\begin{array}{l}\text { CCC } \\
5000\end{array}$ & GA 50 GA 200 \\
\hline $\begin{array}{l}\text { Matière sèche totale/ } \\
\text { plante (g) (MST) }\end{array}$ & 256 & 312 & 251 & 470 & 280 \\
\hline $\begin{array}{l}\text { M.S. des gousses/plantes } \\
\text { en \%\% de MST }\end{array}$ & 37 & 52 & 61 & 15 & 0,3 \\
\hline $\begin{array}{l}\text { M.S. du tubercule et } \\
\text { racines/plantes en } \% \\
\text { de MST }\end{array}$ & 42 & 27 & 16 & 64 & 71,7 \\
\hline $\begin{array}{l}\text { M.S. tiges + feuilles/ } \\
\text { plante en \% de MST }\end{array}$ & 21 & 21 & 23 & 21 & 28 \\
\hline \hline
\end{tabular}

GA 200) parallèlement à un étalement de la floraison et à l'avortement des fleurs.

On peut donc en déduire que les substances de croissance ne modifient pas la part de matière sèche affectée aux organes tiges et feuilles mais opèrent une redistribution des matières entre les puits aérien (organes reproducteurs) et souterrain (tubercule).

\section{DISCUSSION - CONCLUSION}

Le $P$. erosus, dans les conditions environnementales des Antilles, présente un port déterminé lorsque la croissance et le développement se déroulent en jours courts $(12 \mathrm{~h})$ et un port indéterminé en jours croissants ou longs (entre $12 \mathrm{~h}$ et $13 \mathrm{~h}$ ). L'héméropériode maximale aux Antilles est 13 heures. La floraison n'intervient qu'en jours décroissants ou courts.

Ce comportement rencontré chez le Pachyrhizus est analogue à celui décrit chez d'autres plantes dont la croissance et le développement sont influencés par la photopériode. Il peut être expliqué par un niveau plus élevé de substances de type gibbérelline dans les plantes soumises aux conditions de jours longs que dans les plantes ayant subi des conditions de jours courts (RAILTON \& WAREING, 1973). La stimulation d'une forte croissance obtenue en jours courts peut être considérée comme l'effet de l'apport de l'acide gibbérellique dont le niveau de la forme endogène serait faible ou nul.

GA favorise l'allongement des entre-nœuds et l'émission des feuilles. La dose apportée par GA 50 dans nos conditions s'approcherait de celle qui sauvegarderait à la fois la croissance, la formation des gousses et du tubercule alors que celle du GA 200 serait fortement inhibitrice à la fois de la floraison et de la nouaison.

Le CCC (1 000 et 5000 p.p.m.) ne supprime pas la croissance végétative de Pachyrhizus en jours courts. On peut dire que cette légumineuse est moins sensible au CCC que la pomme de terre chez laquelle de nombreux auteurs (DIGBY \& DYSON, 1973 ; El-AB et al., 1980) ont montré que les doses de 1000 et de 2000 p.p.m. inhibaient la croissance. La suppression de la croissance végétative est expliquée par l'aptitude du CCC à inhiber la biosynthèse de la gibbérelline (DENNIS et al., 1965 ; PALEG et al., 1965 ; LANG, 1970) et à stimuler la destruction de l'auxine (HALEVY, 1963 ; HeIDE, 1969).

Au niveau de la floraison et de la fructification l'effet favorable du CCC sur ces deux processus a été manifeste. Les rendements en gousses et fruits les plus élevés ont été obtenus en présence de CCC et les plus faibles en présence de GA 200 mais la floraison et la fructification sont moins perturbées avec GA 50. En ce qui concerne le $\mathrm{CCC}$, cet effet favorable à la fructification peut être rapproché des résultats de PROKHORCHIK \& MASHTOKOV (1972) cité dans EL-ABD et al., 1980 qui rapportent que les doses moyennes de CCC améliorent le rendement sans que cela affecte de façon significative la croissance de la partie aérienne. Les auteurs expliquent ce résultat par le fait que les traitements au CCC augmentent la teneur en chlorophylle $a$ et $b$, en caroténoïdes, l'intensité photosynthétique et la phosphorylation cyclique, donc tous les facteurs susceptibles d'augmenter le pool des assimilats. L'effet de l'acide gibbérellique sur la floraison ou la fructification peut être favorable ou défavorable suivant les espèces et les doses utilisées (SACHS \& HACKETT, 1969, 1977). Si la dose de 50 p.p.m. n'est pas encore préjudiciable à ces deux processus chez le Pachyrhizus, celle de 200 p.p.m. en est inhibitrice.

Bien que l'induction de la tubérisation puisse se faire à n'importe quel moment de l'année sous nos conditions climatiques, le remplissage actif des tubercules s'obtient en jours courts et est accéléré après l'induction florale. La compétition qui s'établit entre les deux puits (gousses d'une part et tubercule d'autre part) se traduit par un ralentissement ou une interruption temporaire du grossissement du tubercule, les assimilats étant préférentiellement orientés vers les gousses et les fruits. En présence du CCC on observe une inhibition particlle du grossissement du tubercule aux doses utilisées. Si on considère uniquement la production du tubercule, ce résultat est différent de celui de RADWAN et al. (1971) sur la pomme de terre qui rapporte une augmentation du rendement en tubercule grâce à l'effet stimulant du CCC sur la production de la matière siche.

Habituellement le CCC est utilisé en conditions non inductives pour promouvoir la tubérisation et cet effet est expliqué par son action antigibbérellique (DIGBY \& DYSON, 1973 ; KUMAR \& WAREING, 1975). En conditions de jours courts où le niveau de substances de type antigibbérellique est élevé, l'apport de CCC exogène peut conduire encore à l'augmentation de ce niveau ou à une réduction du niveau déjà faible de la gibbérelline dont on sait qu'une certaine teneur est non seulement nécessaire à l'induction de la tubérisation mais aussi au grossissement du tubercule (HAMMES \& NEL, 1975). Ainsi pourraient s'expliquer à la fois l'effet inhibiteur du CCC et l'effet favorable de l'acide gibbérellique exogène sur le grossissement du tubercule du Pachyrhizus en jours courts.

L'acide gibbérellique aux doses de 50 p.p.m. et de 200 p.p.m. favorise le grossissement du tubercule chez 
cette plante. Cet effet est important à souligner car cette phytohormone est connue pour avoir un rôle inhibiteur sur la formation et le développement des organes de réserves des plantes à tubercule (OKAZAWA, 1960 ; KUMAR \& WAREING, 1974 ; KUMAR \& BAIJAL, 1979) à cause de son effet sur la distribution des sucres dont la migration vers le tubercule est perturbée (LOVELL \& BOOTH, 1967).

Jusqu'ici les facteurs de croissance comme le CCC et l'acide gibbérellique ont été utilisés sur les plantes produisant un seul organe de réserves : graines ou tubercules. Les résultats rapportés par les différents auteurs sont discutés en terme de relations entre une source qui est constituée par les feuilles (organes exportateurs) et un seul puits. Les facteurs de croissance interviennent alors pour limiter les capacités d'exportation de la source ou celles d'importation du puits. Dans le cas du Pachyrhizus présentant une seule source mais deux puits, le problème se pose aussi en terme de compétition entre ces 2 puits au niveau de la répartition des assimilats. Ces mêmes facteurs n'ont pas modifié fondamentalement le rapport des matières sèches accumulées dans la partie végétative et dans les puits mais ils ont affecté essentiellement la répartition des matières de réserve entre les gousses et le tubercule. Ainsi le CCC, en élevant le nombre de gousses a augmenté la taille de ce puits qui est devenu très compétitif au détriment du tubercule ; le GA en réduisant ou en supprimant le puits constitué par les gousses a favorisé un meilleur remplissage du tubercule. Un dernier puits, non négligeable dans l'utilisation des assimilats, est constitué par les nodosités dont le développement a été très faible et négligeable dans cette étude. Un travail récent portant sur les effets de ces facteurs sur le développement et le fonctionnement des nodosités montre que l'acide gibbérellique réduit le nombre de nodosités formées mais n'affecte pas l'activité spécifique (GOJON, communication personnelle).

Reçu le ler août 1986. Accepté le 5 juillet 1987.

\section{RÉFÉRENCES BIBLIOGRAPHIQUES}

Cortes B. P., 1970. Cultivo de la jicama, Novelas horticolas, 15, 31 34.

Delecolle R., Simon J. C., 1976. Quelques méthodes statistiques appliquées à l'étude des incidences agronomiques d'un aménagement. Note interne M76-3.

Dennis D. T., Upper C. D., West G. A., 1965. An enzymatic set inhibition of gibberellin biosynthesis by Amo 1618 and other plant growth retardants. Plant Physiol., 40, 948.

Digby Y., Dyson P. W., 1973. A comparison of the effects of photoperiod and a growth retardant $\mathrm{CCC}$ on the control of stem extension in potato. Potato Res., 16, 159-167.

El-Abd S. O., El-Beltagy M. S., Abou-Hussein M. R., ElBeltagy A. S., Maksoud M. A., 1980. Effect of some growth regulators on growth and development of potato plants. Egypt. $J$. Hort., 7, 169-180.

Halevy A. H., 1963. Interaction of growth retarding compounds and gibberellin on indole acetic acid oxidase and peroxidase of cucumber seedlings. Plant Physiol., 38, 731.

Hammes P. S., Nel P. C., 1975. Control mechanisms in the tuberization process. Potaio Res., 18, 262-272.

Hansberry R., Clausen R. T., Norton L. B., 1945. Variations in the chemical composition and insecticidal properties of the yam bean (Pachyrrhizus). J. Agric. Res., 74, 55-64.

Heide O. M., 1969. Interaction of growth retardants and temperature in growth, flowering regeneration and auxin activity of Bigonia $\times$ Cheimantha Everett. Physiol. Plant., 22, 1001.

Kumar P., Baijal B. D., 1979. The role of various growth regula tors on plant growth and development of potato (Solanum tubero sum L.). I. General growth reponses of plant. Agra Un. J. Res., 28, 23-30.

Kumar D., Wareing P. F., 1975. Studies on tuberization of Solanum andigena. 2. Growth hormones and tuberization. New Phytol., 73, $833-840$

Lamaze T., Vansuyt G., Zinsou C., 1985. Répartition des formes d'azote et catabolisme des purines chez le dolique tubéreux, Pachy. rhizus erosus (Urban). Agronomie, 5, 933-938.

Lang A.. 1970. Gibberellins, structure and metabolism. Ann. Rev. Plant Physiol., 21, 537-570.

Iovell P. H., Booth A.. 1967. Effects of gibberellic acid on growth, tuber formation and carbohvdrate distribution in Solanum. New Phytol., 66, 525-537.
Marta Evans I., Boulter D., Eaglesham A. R. J., Dart P. J., 1977 Protein content and protein quality of tuberous roots of some legumes determined by chemical method. Qual. Plant. Plant Foods Hum. Nutr., 27, 275-285.

Okazawa Y., 1960. Studies on the relation between the tuber formation of potato and its natural gibberellin content. Proc. Crop Sci. Soc. Japan., 29, 121-124.

Okigbo B. N., 1973. Introducing the yam bean Sphenostylis stenocarpa (Hochst. ex A. Rich) Hams. In proceedings of the first IITA grain legume improvement workshop, 29 oct.-2 nov. 1973. Ibadan pp. 224-238. International Institute of Tropical Agriculture. Ibadan. Nigeria.

Paleg L., Kende H., Ninemann H., Lang A., 1965. Physiological effect of gibberellic acid. VII. Growth retardants on barley enciosperm. Plant Physiol., 40, 165.

Radwan A. A., El-Fouly M. M., Garas N. A., 1971. Retarding stem elongation and stimulating dry matter production and yield of potato with chlormequat chloride (CCC). Potato Res., 14, 173-180.

Railton I. D.. Wareing P. F., 1973. Effects of daylength on endogenous gibberellins in leaves of Solanum andigena. I. Changes in levels of tree acidic gibberellin like substances. Plant Physiol. 28, 88-94.

Sachs R. M. Hackett W. P., 1969. Control of vegetative and reproductive development in seed plants. Hort. Sci., 4, 103-107.

Sachs R. M., Hackett W. P., 1977. Chemical control of flowering. Acta Hortic., 68, 29-49.

Schroeder C. A.. 1967. The jicama. a rootcrop from Mexico. University of California, 65-71.

Srivastava G. S., Shukla D. S., Awaasthi D. N., 1973. We can grow sankalu in the plains of Uttar Pradesh. Indian Farming, 33, 3-9.

Zepeda A. H., 1971. Efecto de desfloracion de la jicama (Pachyrhizus erosus) sobre el rendimiento. Dept. Hort. INIA SAG. CIAB Celoya GTO Mexico. 1, 146-151.

Zinsou C., Vansuyt G., Venthou-Dumaine A., 1987. Croissance et développement du Pachyrhizus erosus (Urban). II. Effets de l'acide gibbérellique et du chlorure de chlorocholine sur la composition glucidique du tubercule. Agronomie, 7 (10). 